Draft Version June 21, 2013

Preprint typeset using $\mathrm{L}^{A} \mathrm{~T}_{\mathrm{E}} \mathrm{X}$ style emulateapj v. 08/22/09

\title{
FCC046: A CANDIDATE GASEOUS POLAR RING DWARF ELLIPTICAL GALAXY IN THE FORNAX CLUSTER
}

\author{
S. De Rijcke ${ }^{1}$, P. Buyle ${ }^{1}$, M. Koleva ${ }^{1}$ \\ Draft version June 21, 2013
}

\begin{abstract}
FCC046 is a Fornax Cluster dwarf elliptical galaxy. Optical observations have shown that this galaxy, besides an old and metal-poor stellar population, also contains a very young centrally concentrated population and is actively forming stars, albeit at a very low level.

Here, we report on $21 \mathrm{~cm}$ observations of FCC046 with the Australia Telescope Compact Array (ATCA) which we conducted in the course of a small survey of Fornax Cluster early-type dwarf galaxies. We have discovered $\mathrm{a} \sim 10^{7} \mathrm{M}_{\odot}$ Hi cloud surrounding FCC046. We show that the presence of this significant gas reservoir offers a concise explanation for this galaxy's optical morphological and kinematical properties.

Surprisingly, the Hi gas, as evidenced by its morphology and its rotational motion around the galaxy's optical major axis, is kinematically decoupled from the galaxy's stellar body. This is the first time such a ring of gaseous material in minor-axis rotation is discovered around a dwarf galaxy.

Subject headings: galaxies: dwarf — galaxies: evolution — galaxies: clusters: individual (Fornax Cluster) - galaxies: stellar content
\end{abstract}

\section{INTRODUCTION}

FCC046 was included in the Fornax Cluster Catalog by Ferguson (1989). It was classified as a dE4, i.e. as a rather flattened dwarf elliptical galaxy, with a total B-band apparent magnitude $m_{B}=15.99 \mathrm{mag}$ and a half-light radius of $R_{e}=6.7^{\prime \prime}$. We adopt a distance of $20.3 \mathrm{Mpc}$ (Jerjen 2003) for the distance to the Fornax Cluster, yielding a total absolute magnitude of $M_{B}=$ $-15.55 \mathrm{mag}$ and a half-light radius of $R_{e}=660$ parsec for FCC046 (De Rijcke et al. 2005). Using VLT photometry and spectroscopy, De Riicke \& Debattista (2004) showed that FCC046, despite its pronounced flattening, has zero net stellar rotation about neither the projected major or the minor axis. It also has a very pronounced nucleus which is resolved from the ground. The nucleus' most striking feature is that, surprisingly, it is off-center with respect to the galaxy's outer isophotes by almost 1 arcsec. This displacement has been interpreted as a consequence of the counter-rotation instability.

\section{EXISTING OPTICAL OBSERVATIONS}

Observations with the FLAIR-II spectrograph on the UK Schmidt Telescope by Drinkwater et al. (2001) have shown that FCC046 has $\mathrm{H} \alpha$ emission, with an equivalent width of $2.1 \AA$ over a fibre 6.7 arcsec accross centered on the galaxy. The presence of ionized gas was interpreted as evidence for star formation, casting doubt on its classification as a "true" dwarf elliptical.

This has later been confirmed by De Rijcke et al. (2003), who showed, based on VLT photometry, that FCC046 has strong positive color gradients and that its nucleus is surrounded by 6 sources of $\mathrm{H} \alpha$ emission. The largest of the emission sources have properties (diameters and $\mathrm{H} \alpha+[\mathrm{NII}]$ luminosities) similar to those of supernova remnants. The smaller ones could be HiI regions or

\footnotetext{
${ }^{1}$ Ghent University, Dept. Physics \& Astronomy, Krijgslaan 281, S9, B-9000, Ghent, Belgium
}

nebulae around Wolf-Rayet stars. Blindly applying the heuristic relation between the star-formation rate and the $\mathrm{H} \alpha$ emission line's equivalent width (Kennicutt 1992) leads to a star-formation rate of $\sim 10^{-3} \mathrm{M}_{\odot} /$ year. The colour gradient also suggests the occurrence of centrally concentrated recent star formation in FCC046.

Applying the full-spectrum fitting code ULySS to an optical VLT spectrum of FCC046, Koleva et al. (2009) showed that the SSP-equivalent age of the stellar population increases from less than 1 Gyr near the center to $\sim 5$ Gyr beyond $1 \mathrm{kpc}$ (see fig. 2 of Koleva et al. (2009)). At the same time, the mean luminosity weighted metallicity, quantified by the SSP-equivalent $[\mathrm{Fe} / \mathrm{H}]$, increases very slightly from a central value of $[\mathrm{Fe} / \mathrm{H}] \sim-1.0$ dex to $[\mathrm{Fe} / \mathrm{H}] \sim-0.8$ dex at $1 R_{e}$.

By fitting a weighted sum of several SSPs to the spectra one can reconstruct a star-formation history (Le Borgne et al. 2004). The number of age bins used in this fit was steadily increased until the goodness-of-fit stopped increasing significantly. The bounds of the bins were varied to ensure that the age and metallicity of each $\mathrm{SSP}$ were well within their respective bounds (e.g. if the age of an SSP coincided with the lower age bound of its bin, this bound was lowered).

In Table 1, we present the results from fits to spectra summed within the inner seeing disk (1 arcsec accross) and within one $R_{e}$ (excluding the inner seeing disk). Within the inner 1 arcsecond, young stars make up $59 \%$ of the light; within the inner $1 \mathrm{R}_{e}$, they constitute $47 \%$ of the light, with about $10 \%$ of newborn stars. Apparently, star formation picked up about $300 \mathrm{Myr}$ ago in the body of the galaxy while the increase in the inner 1 arcsecond happened only $\sim 70 \mathrm{Myr}$ ago. Clearly, the positive age gradient observed in FCC046 is caused by the presence of centralised ongoing star formation, resulting in a very young stellar population whose fractional light contribution decreases with radius.

Dividing the stellar mass in each age bin by its age 

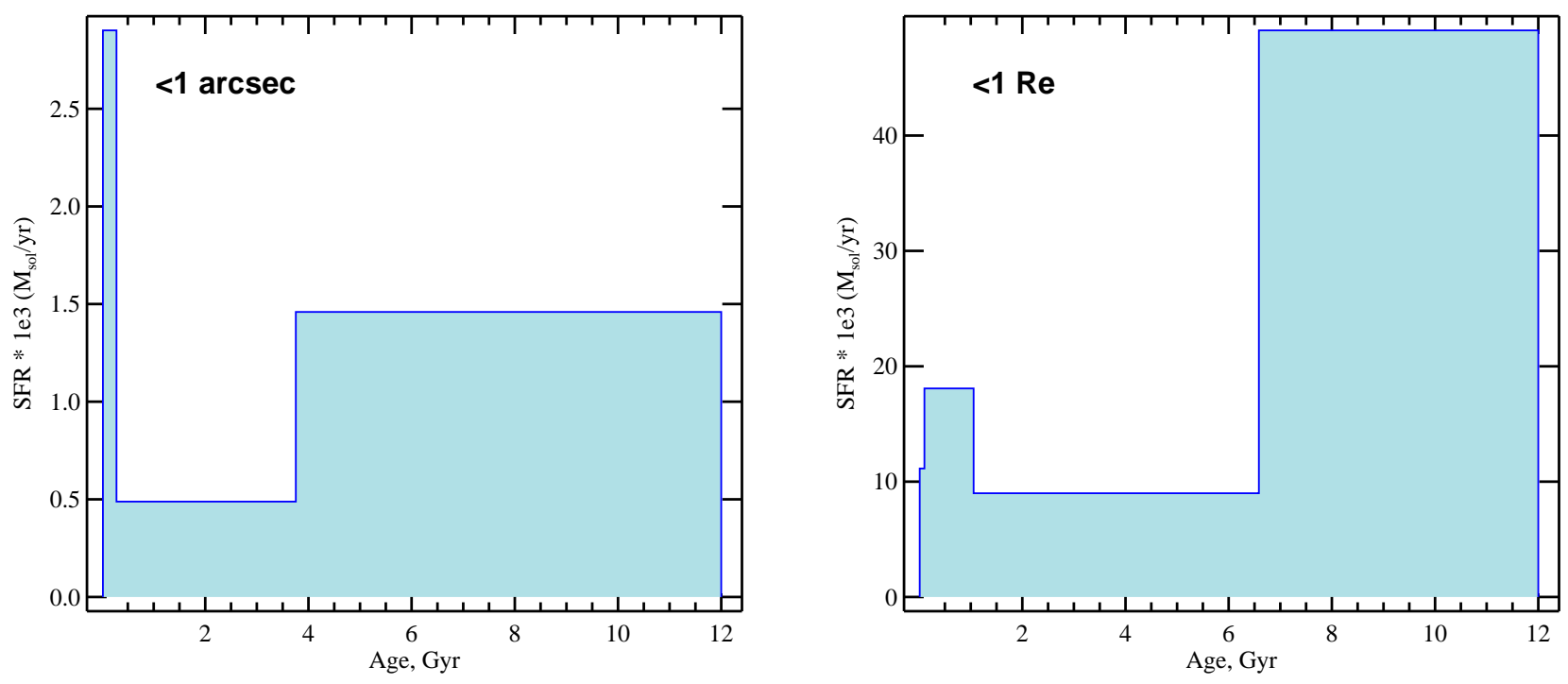

FIG. 1. - Left panel: the star-formation rate as a function of stellar population age as derived from the stacked spectra inside 1 arcsec. Right panel: the same but derived from stacked spectra between 1 arcsec and $1 R_{e}(6.7$ arcsec $)$. This figure is based on a careful re-analysis of the data presented in Koleva et al. (2009).

width yields the mean star-formation rate in each bin, shown in Fig. 11. The central 1 arcsecond wide disk has a B-band luminosity of $L_{B}\left(1^{\prime \prime}\right)=1.0 \times 10^{7} L_{B, \odot}$; the inner $1 \mathrm{R}_{e}$ (excluding the inner disk) has a luminosity $L_{B}\left(1 R_{e}\right)=1.2 \times 10^{8} L_{B, \odot}$. The corresponding masses for the stellar populations inside the inner 1 arcsec and inside $1 R_{e}$, derived with the Pegase.HR evolutionary code for a Salpeter IMF using the SFH reconstruction of ULySS, are $\mathrm{M}\left(1^{\prime \prime}\right)=1.5 \times 10^{7} \mathrm{M}_{\odot}$ and $\mathrm{M}\left(1 R_{e}\right)=3.3 \times 10^{8} \mathrm{M}_{\odot}$. We normalize the SFR such that the time-integral over the star-formation history in each radial bin yields the correct total mass. The recent star-formation event appears to have been very pronounced in the inner regions. There, the star-formation rate increased by over a factor of 5 compared with the mean rate over the previous $\sim 4$ Gyr, although the time resolution of the SFH does not allow us to pinpoint other similar bursts older than $\sim 1$ Gyr.

The oldest stars (age $>3 \mathrm{Gyr}$ ) have metallicities in the range $[\mathrm{Fe} / \mathrm{H}] \sim-2$ to -1 throughout the whole galaxy. Judging from Table 1, the young and intermediate-age stellar populations have metallicities consistent with solar while the 12 Gyr old population has a higher metallicity in the outer radial bin $([\mathrm{Fe} / \mathrm{H}] \sim-1.5$ versus $[\mathrm{Fe} / \mathrm{H}] \sim$ -2.3 inside the inner 1 arcsec). This appears to be the cause for the slight radial increase of the SSP-equivalent metallicity. The metallicity of the very youngest stellar populations is rather uncertain since their massive stars, which dominate their optical light output, have no conspicuous metallicity-sensitive absorption features. However, there appears to be a tendency for the very youngest stellar populations to have metalicities below solar.

Within the inner 1 arcsecond, the nucleus contributes $\sim 80 \%$ of the light while $\sim 20 \%$ comes from the galaxy's main body, adopting the latter's Sérsic profile parameters from Koleva et al. (2009). If we assume that the light fraction of $11 \%$ contributed by very young stars is typical for the galaxy's body (see Table 1) then the $59 \%$ of light from the central 1 arcsecond coming from
TABLE 1

Stellar POPULATIONS INSIDE THE INNER 1 ARCSECOND (TOP) AND INSIDE $1 R_{e}$ (ВOTTOM)

\begin{tabular}{|c|c|c|}
\hline \multicolumn{3}{|l|}{ Inside 1 arcsecond } \\
\hline Age & light fraction & {$[\mathrm{Fe} / \mathrm{H}]$} \\
\hline $67 \pm 5 \mathrm{Myr}$ & $59 \%$ & $-0.11 \pm 0.07$ \\
\hline $1176 \pm 352 \mathrm{Myr}$ & $14 \%$ & $-0.01 \pm 0.27$ \\
\hline $12000 \mathrm{Myr}$ (fixed) & $27 \%$ & $-2.27 \pm 0.13$ \\
\hline \multicolumn{3}{|l|}{ Inside $1 R_{e}$} \\
\hline Age & light fraction & {$[\mathrm{Fe} / \mathrm{H}]$} \\
\hline $34 \pm 10 \mathrm{Myr}$ & $11 \%$ & $-0.80 \pm 0.45$ \\
\hline $309 \pm 51 \mathrm{Myr}$ & $36 \%$ & $0.16 \pm 0.12$ \\
\hline $3613 \pm 1857 \mathrm{Myr}$ & $8 \%$ & $0.27 \pm 0.38$ \\
\hline $12000 \mathrm{Myr}$ (fixed) & $45 \%$ & $-1.46 \pm 0.16$ \\
\hline
\end{tabular}

very young stars, with ages below $100 \mathrm{Myr}$, translates into a nucleus whose youngest stellar populations contribute $\sim 70 \%$ of its light. The mass of the nucleus is $M_{\text {nuc }} \approx 8 \times 10^{6} \mathrm{M}_{\odot}, 7 \%$ of which consists of very young stars. For this estimate, we adopted a $M / L \approx 0.1$ in solar units for a 70 Myr old population from PEGASEHR (Le Borgne et al. 2004). Therefore, most of the mass of the nucleus was in place before the most recent starformation event.

With such a massive nucleus, box orbits are scattered onto tube and chaotic orbits; conservation of angular momentum then requires that direct and retrograde loop orbits be equally populated (Evans \& Collett 1994; Merritt \& Quinlan 1998; Valluri et al. 2010). If this scenario holds, FCC046 would have developed an axially symmetric counter-rotating stellar body, prone to the counter-rotation instability, over the course of only a few crossing times (De Rijcke \& Debattista 2004).

\section{NEW HI OBSERVATIONS}

We used the Australia Telescope Compact Array (ATCA) during February 2006 to search for $21 \mathrm{~cm}$ emission in a small sample of Fornax Cluster dEs. The observations took place during daytime but were not disturbed by solar radio-frequency interference. The ATCA was positioned in the large EW367 configuration with 


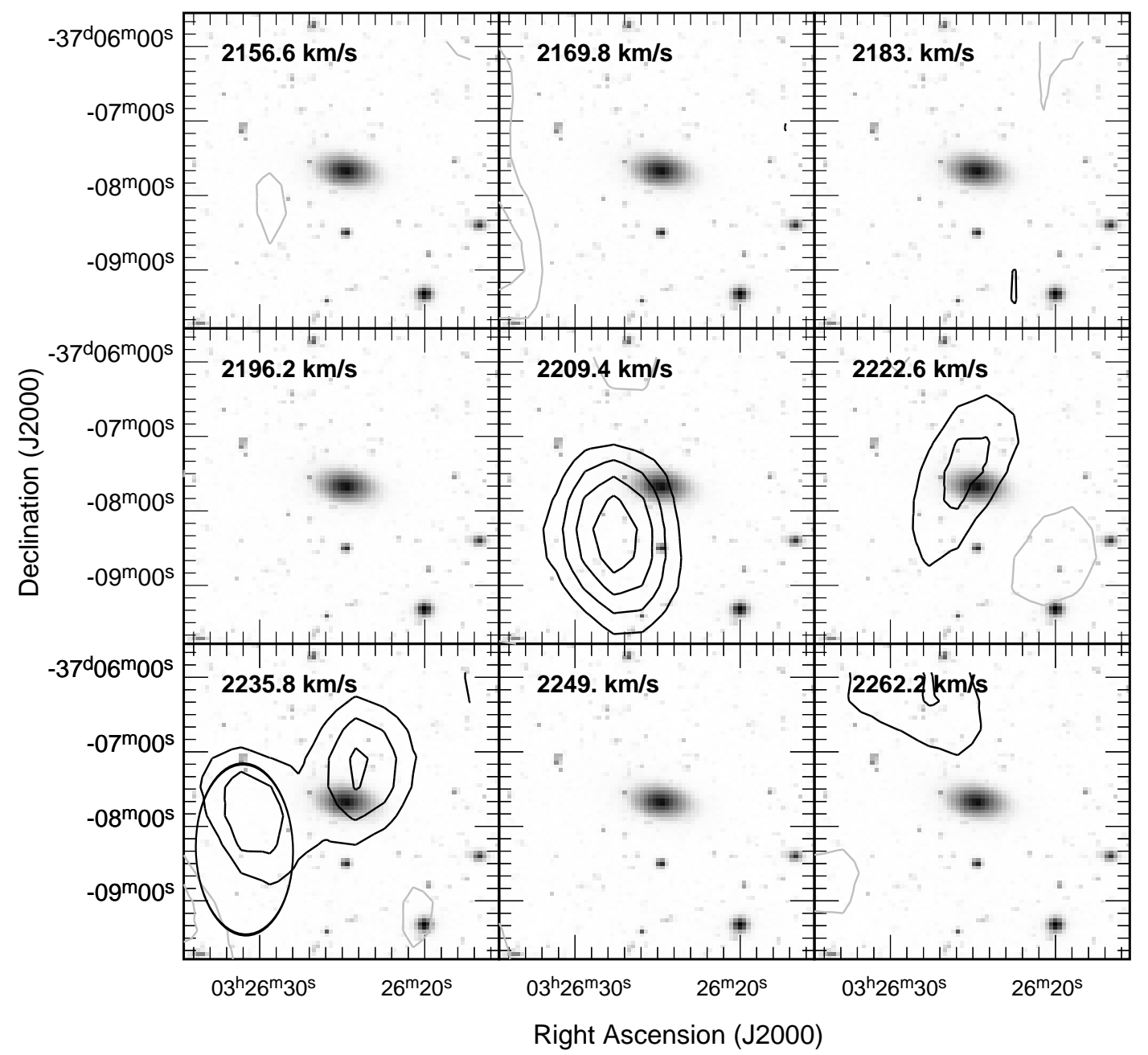

FIG. 2. - $13.2 \mathrm{~km} / \mathrm{s}$ wide channel maps of FCC046. The beam size is indicated by the ellipse in the bottom left panel. Contours are drawn for flux levels of $\pm 2 \sigma, \pm 3 \sigma, \ldots$ with $1 \sigma=3 \mathrm{mJy} /$ beam. Grey contours represent negative flux values. The VLT/FORS2 B-band image in the background is taken from De Rijcke et al. (2003).

baselines ranging from $46 \mathrm{~m}$ up to $4408 \mathrm{~m}$. Due to the typical small velocity widths of dwarf galaxies, we opted for a correlator setup with 256 channels of width 31.25 $\mathrm{kHz}$, giving a total baseband of $8 \mathrm{MHz}$ or roughly 1700 $\mathrm{km} / \mathrm{s}$ at the distance of FCC046. No on-line Hanning smoothing was applied, resulting in a velocity resolution of $6.6 \mathrm{~km} / \mathrm{s}$. The observation was initiated with a calibration on the source 1934-638 which acted as primary calibrator. After the initial calibration, this source was observed for 15 minutes, followed by alternating observations of the phase calibrator 0332-403 (integration of 5 minutes) and of the targeted dwarf galaxy (integration of 40 minutes). The total integration time on each target (including calibration) was $12 \mathrm{~h}$.

The standard data reduction steps (phase, amplitude and bandpass calibration) were performed with the MIRIAD package (Sault, Teuben, \& Wright 1995), the standard ATCA data analysis program. We subtracted the continuum by performing a first-order fit to the visibilities over the line-free channels that were not affected by the edge effects of the band $(300 \mathrm{~km} / \mathrm{s}$ on each edge). The data cubes were created by using natural weighting.
A minimal cleaning of 500 iterations was performed. Our final data cube had a synthesized beam of $147 \times 78 \operatorname{arcsec}^{2}$ and a noise of $3 \mathrm{mJy} / \mathrm{beam}$. These steps resulted in a detection of FCC046 in at least 3 subsequent $6.6 \mathrm{~km} / \mathrm{s}$ channels in the centre of our baseband at a velocity of $2209 \mathrm{~km} / \mathrm{s}$, very close to the optical velocity of FCC046 at $2220 \mathrm{~km} / \mathrm{s}$. Channel maps of FCC046, rebinned to $13.2 \mathrm{~km} / \mathrm{s}$ wide channels, are presented in Fig. 2.

To derive a spectrum we summed the flux within a $4 \times 4 \operatorname{arcmin}^{2}$ box centred on the central radio position of FCC046, which was derived from the total Hi intensity map (see Fig. 3). The global Hi profile shows a double horned spectrum suggesting rotation. A single Gaussian was fitted to the HI profile, giving a velocity linewidth of $52 \mathrm{~km} / \mathrm{s}$ at the 20 per cent level and $34 \mathrm{~km} / \mathrm{s}$ at the 50 per cent level. After correction for broadening and random motions (method of Verheijen \& Sancisi (2001)) we find $W_{20}=43 \mathrm{~km} / \mathrm{s}$ and $W_{50}=32 \mathrm{~km} / \mathrm{s}$.

After summing the channels that contain emission of the galaxy, we found a total velocity-integrated Hi flux density of $0.15 \mathrm{Jy} \mathrm{km} / \mathrm{s}$, resulting in a total estimated HI mass of $1.45 \times 10^{7} \mathrm{M}_{\odot}$. 


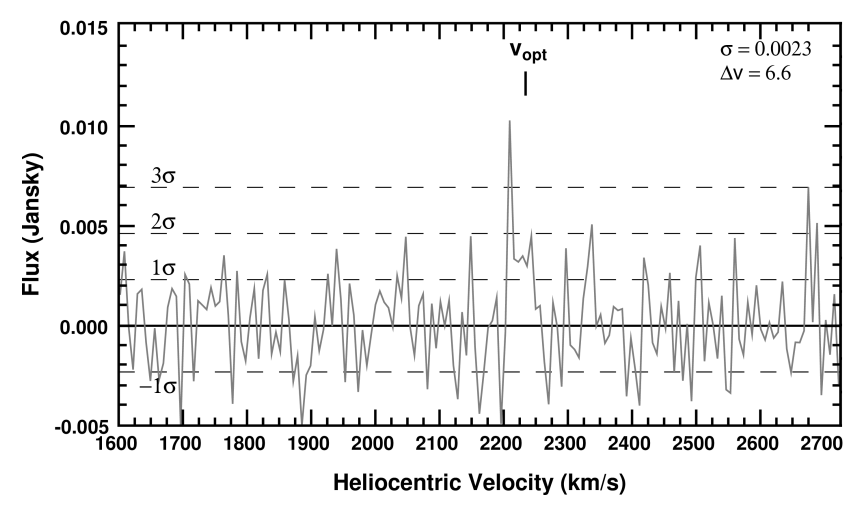

FIG. 3.- Base-line subtracted Hi spectrum of FCC046, extracted from a $4 \times 4$ arcmin $^{2}$ box centred on the central radio position of FCC046. The galaxy's optical velocity is indicated as $v_{\text {opt }}$.

\section{DISCUSSION}

The most striking feature of the $21 \mathrm{~cm}$ emission of FCC046 is that it is elongated in a direction almost perpendicular to the galaxy's stellar body. This is shown clearly in Fig. 4 in which a zeroth-order map of the $21 \mathrm{~cm}$ emission of FCC046 is presented. While the beam size is, admittedly, quite significant, the emission region is clearly resolved and, at FWHM, is almost 1.5 times as large as the beam in the north-south direction. Its east-west extent is not resolved. The first-order moment map shows evidence for a velocity progression from the galaxy's southern side towards the north-west (the velocity increase eastwards of the galaxy is due to a $2 \sigma$ peak in a single channel and is likely to be spurious). If, allowing for the noise level and resolution of our observations, we interpret this as ordered rotation this means that the $\mathrm{HI}$ gas surrounding FCC046 is rotating essentially around the optical galaxy's major axis.

Polar rings, i.e. rings of stars and/or gas and dust orbiting in a plane perpendicular to their host galaxy's equatorial plane, are known in a small fraction of low-mass early-type galaxies (Whitmore et al. 1990; Cox et al. 2006). Cosmological simulations (Macciò et al. 2006) suggest that these galaxies acquire this material through cold accretion of gas filaments. A similar scenario could apply to FCC046. The position of FCC046, at $3.5^{\circ}$ from the cluster center Buyle et al. 2005), places it far outside the X-ray halo of the Fornax Cluster, which does not extend significantly beyond the NGC1399/NGC1404/NGC1387 central region (Jones et al. 1997). This means that any gas accreted onto FCC046 will not immediately be removed by rampressure stripping, keeping it available for star formation. It then stands to reason that FCC046, an "ordinary" dE with a stellar population with a mean age over $\sim 3 \mathrm{Gyr}$, has accreted a few $10^{7} \mathrm{M}_{\odot}$ of gas orbiting roughly perpendicularly to its stellar body. The slightly distorted morphology of the gas may be due to the orbital movement of FCC046 through the Fornax cluster, combined with its weak gravitational field, or by warping. Moreover, if FCC046 has a strongly oblate or prolate gravitational potential, the polar ring gas is expected to rapidly flow inwards where it can be used for star formation (Christodoulou et al. 1992). This inflow, flooding the ring's central hole with gas, would occur on a timescale corresponding to only a few rotation periods. In the case of FCC046, this would amount to a few 100 Myrs which is in line with the observed upturn of the star-formation rate.

Assuming we are indeed observing a gas ring, the observed velocity gradient will be much lower than what could be expected from the galaxy's rotation curve. In the central hole, it is precisely the gas on orbits tangent to the line of sight that is missing. The gas within the ring (back and front side) has much less favorable projections of its rotation velocity onto the line of sight and therefore results in small projected velocities. Only along sufficiently distant lines of sight, those that avoid the central hole, is it possible to observe the full rotation of the gas. However, given the theoretically predicted ongoing warping and disruption of the ring, even there gas is most likely not moving at the local rotation velocity. Along with the severe beam smearing, this helps explains the observed small velocity gradient in the right panel of Fig. 4.

Clearly, the hypothesis that FCC046 has accreted a fresh reservoir of gas that triggered a recent starformation event offers a concise explanation for most of this galaxy's observed properties. Other explanations work far less well. For instance, the observed HI gas could have been expelled by the centralized starformation event, rendering it an outflow rather than an infall. One might expect that, in a flattened dwarf galaxy, such outflows are preferably aligned along the minor axis, as is sometimes observed Mac Low \& Ferrara (1999). However, the recent star-formation rate measured in FCC046 is orders of magnitudes smaller than what is observed in e.g. the star-bursting bipolar outflow dwarf M82, which has a central star-formation rate upwards of $1 \mathrm{M}_{\odot} /$ year (Förster Schreiber, Genzel, Lutz 2003). This makes it unlikely that FCC046 could produce the $G \mathrm{M}_{\text {gal }} \mathrm{M}_{\text {gas }} / 1 \mathrm{kpc} \sim 10^{54} \mathrm{erg}$, the equivalent of over $\sim 1000$ combined supernova explosions (Cloet-Osselaer et al. 2012), needed to lift $\mathrm{M}_{\text {gas }}=1.45 \times$ $10^{7} \mathrm{M}_{\odot}$ of gas out to a distance of $1 \mathrm{kpc}$ in a $\mathrm{M}_{\text {gal }} \sim$ $10^{9} \mathrm{M}_{\odot}$ galaxy. Moreover, realistic simulations of flattened dwarf galaxies fail to produce such collimated outflows if the star-formation activity is not very centrally concentrated (Schroven et al. 2011). Another possibility is that we are seeing gas being removed from FCC046 by ram-pressure stripping (Mori \& Burkert 2000; Marcolini. Brighenti. D'Ercole 2003; Maver et al. 2006). However, FCC046 is very far outside the X-ray halo of the Fornax Cluster, making the stripping scenario highly unlikely.

\section{CONCLUSIONS}

Based on optical observations, FCC046, a dwarf galaxy in the Fornax Cluster, has properties that make it stand out from the general dwarf elliptical galaxy population. All evidence supports the conclusion that $300 \mathrm{Myr}$ ago, the star-formation rate in FCC046 increased dramatically. The strength of this recent star-formation event decreases with radius since these young stars contribue $\sim 60 \%$ of the light within the inner arcsecond (a region largely coinciding with the galaxy's nucleus) while they make up only $47 \%$ of the light within one halflight radius, excluding the central arcsecond. Another odd feature is this galaxy's relatively massive, off-center nucleus. Given the fact that FCC046, despite its signif- 

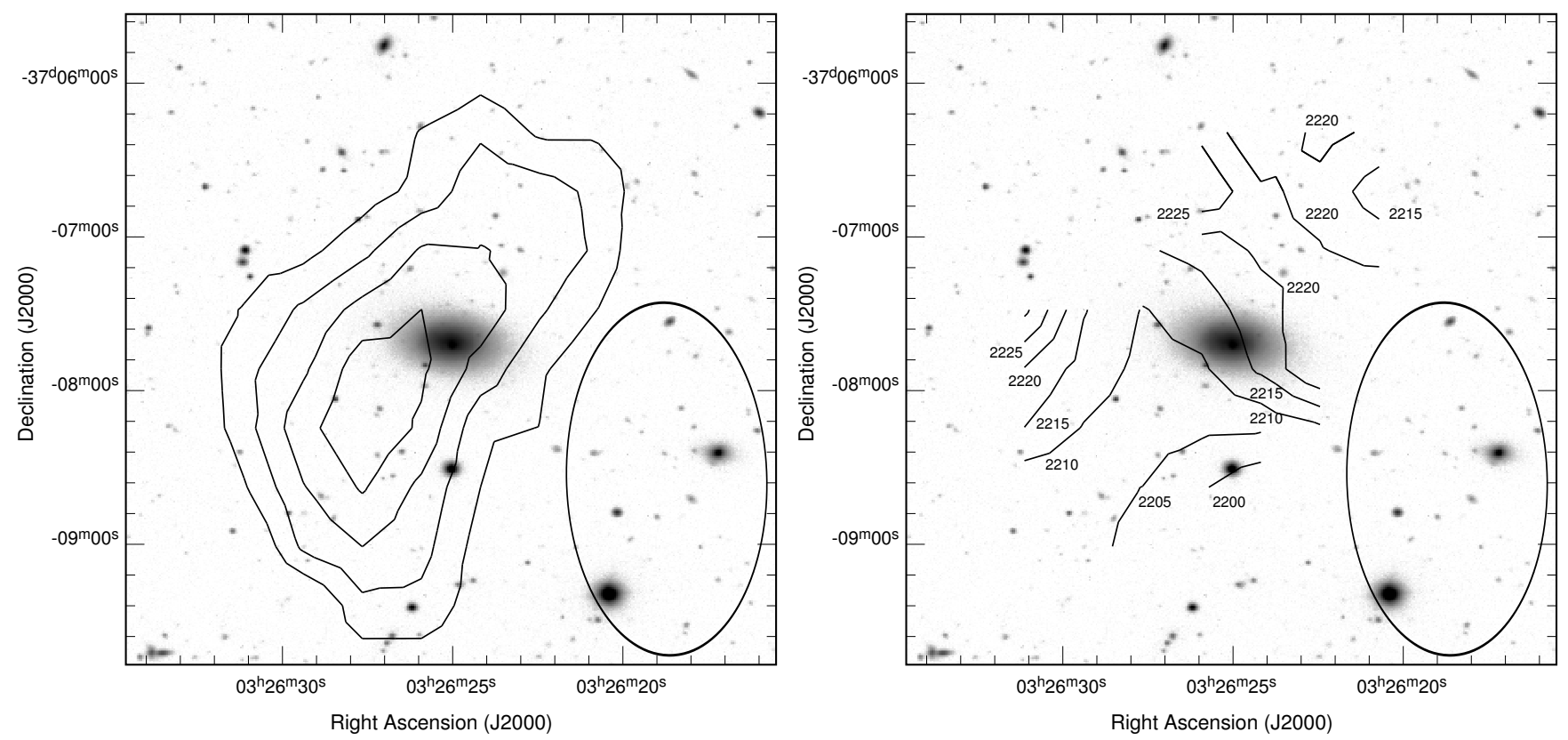

FIG. 4.- Zeroth-order (left) and first-order (right) moment maps of the $21 \mathrm{~cm}$ emission of FCC046. The size and shape of the synthesized beam is indicated in the bottom right corner of each panel. While the beam size is quite significant, the emission is clearly elongated in the direction perpendicular to the galaxy's stellar body. There is also a hint of a velocity progression from the galaxy's southern side towards the north-west. Zeroth-order moment contours are given for the $1 \sigma, 2 \sigma, 3 \sigma$, and $4 \sigma$ levels with $1 \sigma=0.06 \mathrm{Jansky} \mathrm{km} \mathrm{s}^{-1} \mathrm{beam}^{-1}$. The first-order moment map is shown only above the $2 \sigma$ level.

icant flattening, has zero net rotation it is possible that the well-known counter-rotation instability is responsible for driving the nucleus off-center.

In this paper, we have presented Hi observations with the ATCA of FCC046 that may offer a concise explanation for its optical properties. We have discovered $\mathrm{a} \sim 10^{7} \mathrm{M}_{\odot}$ HI cloud surrounding FCC046 which, as evidenced by its morphology and its rotational motion around the galaxy's optical major axis, is kinematically decoupled from FCC046's stellar body. It seems plausible that this gas reservoir has been accreted by FCC046 on a highly inclined orbit and, as it flows to the center of the gravitational well, fuels centrally concentrated star formation.

\section{REFERENCES}

Buyle, P., De Rijcke, S., Michielsen, D., Baes, M., Dejonghe, H., 2005, MNRAS, 360, 853-858

Christodoulou D. M., Katz N., Rix H.-W., Habe A., 1992, ApJ, 395, 113-118

Cloet-Osselaer A., De Rijcke S., Schroyen J., Dury V., 2012, MNRAS, 423, 735-745

Cox, A. L., Sparke, L. S., van Moorsel, G., 2006, AJ, 131, 828-836

De Rijcke, S., Zeilinger, W. W., Dejonghe, H., Hau, G. K. T., 2003, MNRAS, 339, 225-234

De Rijcke, S., Michielsen, D., Dejonghe, H., Zeilinger, W. W., Hau, G. K. T., 2005, A\&A, 438, 491-505

De Rijcke, S. \& Debattista, V. P., 2004, ApJl, 1, L25-L28

Drinkwater, M. J., Gregg, M. D., Holman, B. A., Brown, M. J. I., 2001, MNRAS, 326, 1076-1094

Evans, N. W., \& Collett, J. L., 1994, ApJ, 420, L67-L70

Ferguson, H. C., 1989, AJ, 98, 367-418

Förster Schreiber N. M., Genzel R., Lutz D., 2003, ApJ, 599, 193-217

Ho, N., Geha M., Munoz R. R. Guhathakurta P., Kalirai J., Gilbert K. M., Tollerud E., Bullock J., Beaton R. L., Majewski S. R., 2012, ApJ, 758, 124-135

Jerjen, H., A\&A, 2003, 398, 63-79

Jones, C., Stern, C., Forman, W., Breen, J., David, L., Tucker, W., Franx, M., 1997, ApJ, 482, 143-155

Kennicutt R. C., 1992, ApJ, 388, 310-327
Koleva, M., De Rijcke, S., Prugniel, Ph., Zeilinger, W. W., Michielsen, D., 2009, MNRAS, 396, 2133-2151

Le Borgne D., Rocca-Volmerange B., Prugniel P., Lanon A., Fioc M., Soubiran C., 2004, A\&A, 425, 881-897

Macciò, A. V., Moore, B., Stadel, J., 2006, ApJL, 626, L25-L28

Mac Low, M. \& Ferrara, A., 1999, ApJ, 513, 142-155

Marcolini, A., Brighenti, F., D'Ercole, A., 2003, MNRAS, 345, 1329-1339

Mayer, L., Mastropietro, C., Wadsley, J., Stadel, J., Moore, B., 2006, MNRAS, 369, 1021-1038

Merritt, D., \& Quinlan, G. D. 1998, ApJ, 498, 625

Mori, M. \& Burkert, A., 2000, ApJ, 538, 559-568

Sault, R. J., Teuben, P. J., \& Wright, M. C. H. 1995, in ASP Conf. Ser. 77, Astronomical Data Analysis Software and Systems IV, ed. R. A. Shaw, H. E. Payne, \& J. J. E. Hayes (San Francisco: ASP), 433

Schroyen J., de Rijcke S., Valcke S., Cloet-Osselaer A., Dejonghe H., 2011, MNRAS, 416, 601

Valluri, M., Debattista, V. P., Quinn, T., Moore, B., 2010, MNRAS, 403, 525-544

Verheijen M. A. W. \& Sancisi R., 2001, A\&A, 370, 765

Whitmore B. C., Lucas R. A., McElroy D. B., Steiman-Cameron

T. Y., Sackett P. D., Olling R P., 1990, AJ, 100, 1489-1522 